a marker of primary cilia, is reduced in primary cell culture and in lumbar spinal cord in situ in G93A SOD1 mice. BMC Neurosci. 2011;12:71.

11. Elsaesser R, Paysan J. The sense of smell, its signalling pathways, and the dichotomy of cilia and microvilli in olfactory sensory cells. BMC Neurosci. 2007;8(suppl 3):S1.

12. Kulaga HM, et al. Loss of BBS proteins causes anosmia in humans and defects in olfactory cilia structure and function in the mouse. Nature Genet. 2004;36(9):994-998.

13. Alvarez-Buylla A, Seri B, Doetsch F. Identification of neural stem cells in the adult vertebrate brain. Brain Res Bull. 2002;57(6):751-758.

14. Quinones-Hinojosa A, et al. Cellular composition and cytoarchitecture of the adult human subven- tricular zone: a niche of neural stem cells. J Comp Neurol. 2006;494(3):415-434.

15. Han Y-G, Alvarez-Buylla A. Role of primary cilia in brain development and cancer. Curr Opin Neurobiol. 2010;20(1):58-67

16. Deng W, Aimone JB, Gage FH. New neurons and new memories: how does adult hippocampal neurogenesis affect learning and memory? Nat Rev Neurosci. 2010;11(5):339-350.

17. Bradford J, et al. Mutant huntingtin in glial cells exacerbates neurological symptoms of Huntington disease mice. J Biol Chem. 2010;285(14):10653-10661.

18. Johnston JA, Ward CL, Kopito RR. Aggresomes: a cellular response to misfolded proteins. J Cell Biol. 1998;143(7):1883-1898

19. Knodler A, et al. Coordination of Rab8 and Rab11 in primary ciliogenesis. Proc Natl Acad Sci U S A. 2010; 107(14):6346-6351.

20. del Toro D, et al. Mutant huntingtin impairs postGolgi trafficking to lysosomes by delocalizing optineurin/Rab8 complex from the Golgi apparatus. Mol Biol Cell. 2009;20(5):1478-1492.

21. Li X, et al. Mutant huntingtin impairs vesicle formation from recycling endosomes by interfering with Rab11 activity. Mol Cell Biol. 2009;29(22):6106-6116.

22. Li X, et al. Aberrant Rab11-dependent trafficking of the neuronal glutamate transporter EAAC1 causes oxidative stress and cell death in Huntington's disease. J Neurosci. 2010;30(13):4552-4561.

23. Richards $P$, et al. Dendritic spine loss and neurodegeneration is rescued by Rab11 in models of Huntington's disease. Cell Death Differ. 2011;18(2):191-200.

\title{
Are maternal antiplatelet antibodies a prothrombotic condition leading to miscarriage?
}

\author{
Alvin H. Schmaier \\ Case Western Reserve University, Cleveland, Ohio, USA.
}

\begin{abstract}
Fetal and neonatal alloimmune thrombocytopenia (FNAIT) is a condition characterized by thrombocytopenia in the newborn. If severe, the thrombocytopenia can lead to intracranial hemorrhage. FNAIT arises when maternal antibodies specific for platelet antigens, most commonly $\beta 3$ integrin, cross the placenta and destroy fetal platelets. Surprisingly, few cases of FNAIT are associated with antibodies specific for the platelet antigen GPIb $\alpha$, which is a common target in patients with immune thrombocytopenia. In this issue of the JCI, Li et al. have identified a potential reason for this - they find that in the majority of pregnant mice, anti-GPIb $\alpha$ antibodies enhance platelet activation and accelerate thrombus formation in the placenta and that this leads to miscarriage.
\end{abstract}

Our parents remind us that we should not forget our roots. Hematologists should not forget that the discipline over the last 100 years partly arose to prevent the large number of hemorrhagic deaths associated with labor and delivery (they occurred in $0.25 \%$ of deliveries in 1900 and $0.004 \%$ of deliveries in 2005) (1). Some success has been achieved, for example, in the development of approaches to detect and treat rhesus D hemolytic disease of the newborn: the indirect Coombs test (indirect antiglobulin test), which is used to determine whether a mother has been sensitized by red cell antigens (e.g., the rhesus D antigen $[\mathrm{RhD}]$ ) from a previous pregnancy (2); and the first immunoglobulin therapy, involving the administration of RhD-specific

Conflict of interest: Alvin H. Schmaier receives research support from CSL Behring.

Citation for this article: J Clin Invest. 2011; 121(11):4241-4243. doi:10.1172/JCI60749. antibody to RhD-negative mothers who have been exposed to fetal RhD blood antigens. However, many hematological conditions continue to pose serious problems for individuals who are pregnant, including preeclampsia with hypertension and thrombocytopenia and fetal and neonatal alloimmune thrombocytopenia (FNAIT; also known as neonatal alloimmune thrombocytopenia [NAIT], fetal alloimmune thrombocytopenia [FAIT], or fetal neonatal immune thrombocytopenia [FNIT]).

\section{Maternal antiplatelet antibodies in pregnancy} ing in individuals of European descent in $0.06 \%-0.1 \%$ of live births (3). It is characterized by thrombocytopenia shortly after birth. Twenty percent of women with known prior immune thrombocytopenia will deliver a fetus with a platelet count of less than $50,000 / \mu \mathrm{l}(4)$. Although the thrombocyto-
FNAIT is a relatively rare condition, aris- penia is often mild and the affected neonate remains largely asymptomatic, cases of severe thrombocytopenia carry a high risk of intracranial hemorrhage (5). In fetuses with a platelet count of less than $20,000 / \mu 1$, the incidence of intracerebral hemorrhage is $10 \%-20 \%$, which may lead to neurological impairment or death (5).

FNAIT arises when maternal antibodies specific for platelet antigens cross the placenta and destroy fetal platelets. The maternal antibodies may arise during pregnancy, if fetal platelet antigens are recognized by the maternal immune system as nonself (allogeneic), or be already present in a mother with immune thrombocytopenia. In mothers with immune thrombocytopenia, it is also possible that the situation is not passive, since these individuals may be more sensitive to developing antibodies specific for allogeneic fetal platelet antigens.

Most commonly (in $79 \%-85 \%$ of cases of FNAIT), the pathogenic antibodies are specific for the HPA-1a epitope of $\beta 3$ integrin, a component of one of the major glycoproteins on the surface of platelets $(3,6,7)$. Other targets of the pathogenic maternal alloantibodies include the HPA5 b epitope of GPIa (also known as integrin a2) (in 7\%-10\% of cases) and both HPA-1a and HPA- $5 \mathrm{~b}$ (in $2 \%-7 \%$ of cases) $(3,6,7)$. It is of interest that few cases of FNAIT are associated with antibodies specific for epitopes of GPIb $\alpha$ (HPA-2a/b), a component of another major glycoprotein on the surface 


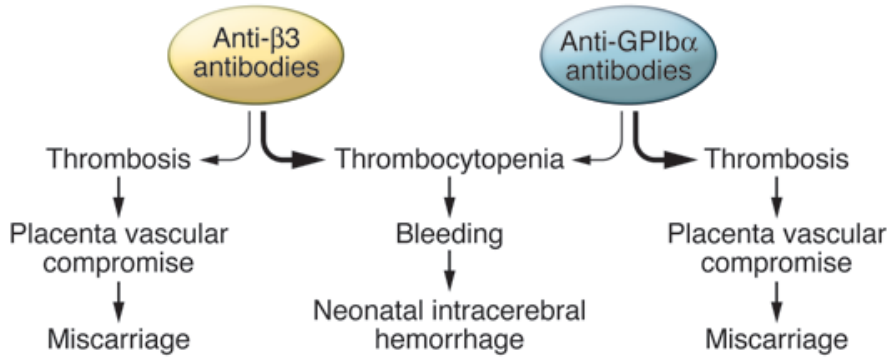

Figure 1

The effects of maternal alloimmune anti- $\beta 3$ antibodies and anti-GPIb $\alpha$ antibodies in pregnancy. Maternal alloimmune antiplatelet antibodies are most commonly directed to the HPA-1a epitope of $\beta 3$ integrin and can cause FNAIT. This entity is most commonly associated with thrombocytopenia and, in severe cases, neonatal intracerebral hemorrhage. FNAIT associated with antiGPIb $\alpha$ antibodies has been rarely recognized. In this issue of the $\mathrm{JCl}$, Li et al. report that in mice with anti-GPIb $\alpha$ antibodies, a high percentage of pregnancies results in placental thrombosis due to increased thrombin formation and that this leads to miscarriage (12). In contrast, mouse anti- $\beta 3$ antibodies were more commonly observed to produce thrombocytopenia and less commonly associated with placental thrombosis and miscarriage. This report suggests that FNAIT may be a cause of thrombosis in addition to hemorrhage and that some antiplatelet antibodies may induce miscarriage.

of platelets, since antibodies specific for GPIb $\alpha$ are present in $20 \%-40 \%$ of patients with immune thrombocytopenia $(8,9)$. Rare cares of FNAIT associated with GPIb $\alpha$ are antibodies to a $\mathrm{C} \rightarrow \mathrm{T}$ polymorphism at nucleotide position 434 , which results in a substitution of threonine (HPA-2a) to methionine (HPA-2b) at amino acid position $145(10,11)$. In this issue of the JCI, Li et al. examine why few cases of FNAIT mediated by antibodies specific for GPIb $\alpha$ are seen (12).

\section{Effect of maternal anti-GPIb $\alpha$ antibodies on pregnancy outcome}

Li et al. generated mice with high levels of antibodies specific for platelet antigens relevant to FNAIT and immune thrombocytopenia (12). Specifically, they immunized mice lacking integrin $\beta 3$ (Itgb3 $3^{-/-}$mice) and mice lacking GPIb $\alpha$ (Gp1ba-/- mice) on a $\mathrm{BALB} / \mathrm{c}$ background with wild-type mouse platelets. Good antibody responses to the missing antigen were mounted in both knockouts. When immunized Itgb3 ${ }^{-1-}$ and Gp1ba/- female mice were bred to wild-type males, it was extremely difficult to obtain thrombocytopenic Gp1ba/- pups. Strikingly, miscarriage occurred in 25 of 30 (83.3\%) pregnancies resulting from breeding

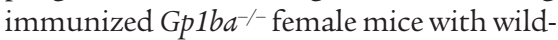
type males versus 10 of 26 (37\%) pregnancies involving immunized Itgb3 $3^{-/}$female mice (12). Further, the miscarriages were observed late in pregnancy (E16.5-E18.5). In the few live births, there was only a $27 \%$ reduction in platelet count in $\mathrm{Gp} 1 \mathrm{ba^{+/- }}$ pups (litter size, $0.4 \pm 0.2$ ) versus a $56 \%$ reduction in platelet count in Itgb $3^{+/-}$pups (litter size, $3.3 \pm 0.8)$. These data indicate that the anti-GPIba antibodies were more likely to cause miscarriage and fetal wastage than neonatal alloimmune thrombocytopenia (Figure 1). The placentas that formed in immunized $G p 1 b a^{-/-}$females versus those in the immunized $\operatorname{Itg} 3^{-/-}$females showed microthrombus formation, increased fibrin deposition, more apoptosis/necrosis on TUNEL assays, and reduced placental circulation at 14.5 days post coitum and before miscarriage. Li et al. further found that anti-GPIb $\alpha$ antisera, unlike anti- $\beta 3$ antisera, enhanced ADP-induced platelet aggregation, JON/A epitope expression (a platelet activation marker showing a complex between $\alpha \operatorname{IIb} \beta 3$ integrins), fibrinogen binding, P-selectin expression, and markedly accelerated thrombus formation on intravital microscopy (12). These data indicate that the anti-GPIb $\alpha$ antibodies induced thrombosis in the placenta (12).

\section{Human antiplatelet antibodies in pregnancy}

The findings of Li et al. that anti-GPIba antibodies induce placental thrombosis leading to miscarriage in pregnant mice are surprising (12). Do they really represent what occurs in humans, or does this report describe a disorder specific to a mouse model? In a human observational study of 385 female subjects lacking the HPA-1a epitope of the $\beta 3$ integrin, $12 \%$ (46 of 385) developed antibodies specific for HPA- 1a (13). Only one fetus among 46 died in utero; 26 HPA-1a-positive babies had persistent antenatal anti-HPA-1a antibodies; 9 had severe thrombocytopenia (platelets less then $10,000 / \mu l) ; 10$ were mildly thrombocytopenic; but 7 had normal platelet counts (13). The outcome observed in this study of human pregnancies, in which antibodies specific for HPA-1a were detected (13), is much better than the $37 \%$ fetal death rate for mouse fetuses exposed to anti- $\beta 3$ antibodies reported by Li et al. (12). Since the incidence of anti-GPIb $\alpha$-mediated FNAIT is rare in humans, miscarriage has not been associated with it. However, one patient with habitual abortion has been reported to have antibodies specific for the GPIb $\alpha$ containing complex (14). Additionally, in a study of 30 pregnancies in 18 women with Bernard-Soulier syndrome (a rare autosomal recessive bleeding disorder that causes a deficiency of the GPIb $\alpha$-containing complex at the platelet surface), there were 6 cases of FNAIT, with one intrauterine death and one neonatal death 6 hours after birth due to an intracranial hemorrhage (15). The question of whether antiGPIb $\alpha$ antibodies really induce miscarriage in humans can be answered only by prospective observational studies in female patients with immune thrombocytopenia and, independently, miscarriages.

\section{Thrombosis risk and miscarriage}

Pregnancy loss is common, with up to $50 \%$ of human conceptions being unsuccessful (16). Hematologists assist obstetricians in investigating the cause of recurrent abortion by performing prothrombotic evaluations. Women with antiphospholipid antibodies have a strong link between thrombophilia and pregnancy loss. Anticardiolipin and anti- $\beta 2$-glycoprotein I antibodies and lupus anticoagulants also are strong risk factors for miscarriage, since these antibodies are prothrombotic themselves (16-18). The inherited thrombophilias (factor $V$ Leiden and prothrombin 20210) and the diseases caused by deficiency of several anticoagulants (antithrombin, protein $\mathrm{C}$, or protein $\mathrm{S}$ ) are associated with early and late recurrent pregnancy loss (16). The work of Li et al. (12) may be a game changer on how we approach women with miscarriages. If it is shown in humans that FNAIT is associated with pregnancy loss, maternal antibodies in immune thrombocytopenia or elicited by exposure to allogeneic fetal platelet antigens will also need to be considered in the evaluation 
of any woman with recurrent abortion. It is surprising that Li et al. observed that embryos in the immunized Itgb3 $3^{-/-}$female mice, which developed anti- $\beta 3$ antibodies, had a $37 \%$ intrauterine death rate. As noted above, human anti-HPA- 1 a antibodies are associated only with a $2 \%$ intrauterine death rate $(12,13)$.

How exactly do the antibodies specific for GPIb $\alpha$ induce thrombosis? Li et al. indicate that the mouse antibodies specific for GPIb $\alpha$ may reduce thrombin binding to its high-affinity receptor, allowing more "free" thrombin to proteolyze fibrinogen and make fibrin (12). One wonders if that is possible, considering the presence of plasma antithrombin and heparin cofactor II and cell membrane protease nexin 1 . How antiGPIb $\alpha$ antibodies potentiated ADP-induced platelet aggregation was not elucidated in the report. It is possible that excess free thrombin binds PAR4 and primes the P2Y12 ADP receptor to be more responsive to ADP (19). Regardless of the mechanism(s), the antibodies specific for GPIb $\alpha$ themselves induced accelerated thrombus formation on intravital microscopy (12), indicating that they have a direct role in leading to vessel thrombosis. However, more investigations are needed to further characterize the precise mechanism(s) by which they lead to placental vessel thrombosis.

\section{Management of maternal anti-GPIb $\alpha$ antibodies}

Until such time as we know whether antibodies specific for GPIb $\alpha$ are prothrombotic in humans and can cause miscarriage, how are we going to treat women who have alloimmune antiplatelet antibodies and miscarry pregnancies? The report by $\mathrm{Li}$ et al. (12) suggests at least two approaches. First, the authors demonstrated that low-dose warfarin therapy ameliorated the miscarriage rate in the immunized $\mathrm{GPIba}^{-/-}$female mice (12). However, the utility of antithrombotics as a measure to prevent miscarriage in patients with inherited thrombophilia is not proven, although their use in patients with antiphospholipid antibodies may be beneficial (16). Further, warfarin itself in the first trimester is con- traindicated in pregnant women because of a possible birth defect risk. An alternative approach is to manage these patients with immunoglobulin therapy with or without corticosteroids, a standard approach for both alloimmune hemolysis and thrombocytopenia during pregnancy. At present, the clinical data are unclear as to whether the addition of corticosteroids to immunoglobulin therapy is better in mothers who have alloimmune antiplatelet antibodies and are at risk of having FNAIT during pregnancy (3). In support of this approach, Li et al. demonstrate that intravenous immunoglobulin (IVIG) and anti-FcRn infusions prevented miscarriage in their mouse model of anti-GPIb $\alpha$-mediated FNAIT (12). Further, the platelet counts were preserved in the neonates, and the number of pups per litter increased. In sum, Li et al. report in this issue of the JCI important work that suggests that maternal alloimmunization by GPIb $\alpha$ platelet antigens uncommonly produces fetal thrombocytopenia with risk for intracranial hemorrhage, but more likely creates a placental prothrombotic state leading to fetal wastage (12) (Figure 1). This notion is a step forward in our understanding of the breadth of hematologic complications associated with FNAIT.

\section{Acknowledgments}

This work was supported in part by grants HL052779, CA076917, AG03661, and HL072033.

Address correspondence to: Alvin H. Schmaier, Case Western Reserve University, University Hospitals Case Medical Group, 10900 Euclid Avenue, WRB2130, Cleveland, Ohio 44106, USA. Phone: 216.368.1172; Fax: 216.368.3014; E-mail: schmaier@case.edu.

1. Hogan MC, et al. Maternal mortality for 181 countries, 1980-2008: a systematic analysis of progress towards Millennium Development Goal 5. Lancet. 2010;375(9726):1609-1623.

2. Coombs RR, Mourant AE, Race RR. A new test for the detection of weak and "incomplete" Rh agglutinins. BrJ Exp Path. 1945;26:225-266.

3. Rayment R, Brunskill SJ, Soothhill PW, Roberts DJ, Bussel JB, Murphy MF. Antenatal interventions for fetomaternal alloimmune thrombocytopenia. Cochrane Database Syst Rev. 2011;(5):CD004226.

4. Samels P, et al. Estimation of the risk of thrombocytopenia in the offspring of pregnant women with presumed immune thrombocytopenic purpura. $N$ Engl J Med. 1990;323(4):229-235.

5. Bussel JB, Zabusky MR, Berkowitz RL, McFarland JG. Fetal alloimmune thrombocytopenia. N EnglJ Med. 1997;337(1):22-26.

6. Davoren A, Curtis BR, Aster RH, McFarland JG. Human platelet antigen-specific alloantibodies implicated in 1162 cases of neonatal alloimmune thrombocytopenia. Transfusion. 2004;44(8):1220-1225.

7. Knight M, et al. The incidence and outcomes of fetomaternal alloimmune thrombocytopenia: a UK national study using three data sources. $\mathrm{Br} \mathrm{J}$ Haematol. 2011;152(4):460-468.

8. He R, Reid DM, Jones CE, Shulman NR. Spectrum of Ig classes, specificities, and titers of serum antiglycoproteins in chronic idiopathic thrombocytopenic purpura. Blood. 1994;83(4):1024-1032.

9. McMillan R. Autoantibodies and autoantigens in chronic immune thrombocytopenic purpura. Semin Hematol. 2000;37(3):239-248.

10. Goldman M, Trudel E, Richard L, Khalife S, Spurll GM. Neonatal alloimmune thrombocytopenia due to anti-HPA-2b (anti-Koa). Immunohematology. 2003;19(2):43-46.

11. Kuijpers RWAM, Faber NM, Cuypers HTM, Ouwehand WH, von dem Borne AEGK. $\mathrm{NH}_{2}$-terminal globular domain of human platelet glycoprotein Iba has a methionine $\mathrm{e}^{145} /$ threonine $^{145}$ amino acid polymorphism, which is associated with the HPA-2(Ko) alloantigens. J Clin Invest. 1992;89(2):381-384.

12. Li C, et al. The maternal immune response to fetal platelet GPIb $\alpha$ causes frequent miscarriage in mice that can be prevented by intravenous IgG and anti-FcRn therapies. J Clin Invest. 2011; 121(11):4537-4547.

13. Williamson LM, et al. The natural history of fetomaternal alloimmunization to the platelet-specific antigen HPA-1a $\left(\mathrm{Pl}^{\mathrm{A} 1}, \mathrm{Zw}^{\mathrm{a}}\right)$ as determined by antenatal screening. Blood. 1998;92(7):2280-2287.

14. Hickstein H, Barz D, Kulz T, Korten G, Muller $\mathrm{H}$, Schmidt R. Protein A immunoadsorption in a pregnant woman with habitual abortion. Transfus Apher Sci. 2002;27(3):259-261.

15. Peitsidis P, Datta T, Pafilis I, Otonmewo O, Tuddenham EG, Kadir RA. Bernard-Soulier syndrome in pregnancy: a systematic review. Haemophilia. 2010;16(4):584-591.

16. Bates SM. Consultative hematology: the pregnant patient pregnancy loss. Hematology Am Soc Hematol Educ Program. 2010;2010:166-172.

17. Alijotas-Reig J, et al. Are anti-Beta2-glycoprotein-I antibodies markers for recurrent pregnancy loss in lupus anticoagulant/anticardiolipin seronegative women? Am J Reprod Immunol. 2008;60(3):229-237.

18. Arad A, Proulle V, Furie RA, Furie BC, Furie B. b2-glycoprotein-1 autoantibodies from patients with antiphospholipid syndrome are sufficient to potentiate arterial thrombus formation in a mouse model. Blood. 2011;117(12):3453-3459.

19. Holinstat M, Voss B, Bilodeau ML, McLaughlin JN, Cleator J, Hamm HE. PAR4, but not PAR1, signals human platelet aggregation via $\mathrm{Ca} 2+$ mobilization and synergistic P2Y12 receptor activation. J Biol Chem. 2006;281(36):26665-26674. 